\title{
USOS PARALELOS DEL INFINITIVO EN LATÍN E INGLÉS: ESTILO INDIRECTO Y COMPLEMENTACIÓN
}

\author{
M.' Cruz Garcta fuentes y Ángeles Ortega Calvo
}

\section{1}

Ciertamente la realidad de una lengua es muy compleja, y ofrece al que la conoce una serie de ángulos desde los que puede ser contemplada y en los que se puede recalar intentando establecer una serie de paralelos y semejanzas con otras lenguas afines o diversas.

Es evidente que cualquier lengua dispone de unas opciones variables de las que se sirve para expresar determinados contenidos, $y$ esas opciones también se dan en otras lenguas, $y$, a veces, tal vez debido a la casualidad, interferencia, influencia, o lógica, se utilizan unas mismas opciones con determinados contenidos verbales en dos o más lenguas.

\section{2}

Pues bien, teniendo como base éste hecho y reflexionando sobre ciertas coincidencias observadas en ambas lenguas, hemos llevado a cabo un trabajo de carácter sincrónico-tipológico en el que, de forma sistemática, hemos analizado la utilización de ciertas construcciones de infinitivo en las que se observan evi- 
dentes paralelos entre la lengua latina y la inglesa, siendo muy difícil llegar a delimitar los conceptos de «interferencia» y «préstamo» en los hechos que vienen a continuación.

Y es que nuestro propósito no es dilucidar aquí si tales semejanzas se deben a una base común indoeuropea, de la que, por otra parte, no existe prácticamente reconstrucción sintáctica; o bien a la influencia que, especialmente del latín sobre el inglés, pudiera haberse producido al tener lugar ciertos contactos entre ambas lenguas, ocasionados prrincipalmente por la traducción de textos ${ }^{1}$.

Es seguro que hubo tales interferencias, pero, dado que apenas tenemos textos - salvo meras inscripciones- en inglés antiguo antes del siglo VII, y puesto que parece no haber diferencias de grado apreciable entre la utilización de estas construcciones en textos anglosajones originales y textos traducidos del latín, no podemos delimitar con exactitud si tales similitudes estructurales se deben a integración de estas formas latinas por parte de el inglés o a un desarrollo paralelo, pero independiente, de cada una de las lenguas. A esta última hipótesis parecería contribuir el hecho de que, si bien el paralelismo al que aludimos es muy consistente, no cubre para las dos lenguas todo el espectro de utilización del infinitivo; a este respecto, el inglés ha desarrollado más aplicaciones que el latín, mientras que, en contrapartida, desconoce algunos usos que en latín son frecuentes (sobre todo, en lo que respecta al estilo indirecto). Por otro lado, lenguas germánicas como el alemán o el neerlandés, que tuvieron también mucha influencia latina, desarrollaron sin embargo un estilo indirecto con utilización de modal y no de estructura infinitivo, mientras que en inglés ambos, en algunos casos, son posibles lo que podría, por su parte, sustentar la primera hipótesis.

1 Para éste y otos aspectos de la influencia latina sobre la lengua inglesa cf., entre otros, Mitchell, Od English Syntax, Oxford: Clarendon Press (2vols.); Callaway, M Jr (1913), The Infinitive in Anglo-Saxon, Washington; ZEITLIN, J., The Accusative with Infinitive and some Kindred constructions in English, Nueva York, 1908; GorRelL, J.H (1985), «Indirect Discourse in Anglo-Saxon», en PMLA, Publications of the Modern Language Association of America, 10; STEWART, A.H. (1976), "The Development of the verb-phrase complement with verbs of physical perception in English: Historical Linguistics as a sourrce of deep structures», en Journal of English Linguistics, 10, 34-53; MACHACEK, J. 8, (1964), «Historical Aspect of the Accusative with Infinitive and the Content Clause in English», en Brno Studies in English, 8, 123-32; VISSER, F.Th., An Historical Syntax of the English Language (3vols.) Leiden, 1963-73. 


\section{I.3.}

Sea como fuere, nosotras no vamos a ocuparnos de tal cuestión, que puede presentarse irresoluble, y, en lo que sigue, nos limitaremos a ofrecer una muestra del idéntico uso que latín e inglés moderno hacen del infinitivo en los campos de la complementación de adjetivos, sustantivos y verbos, oraciones de infinitivo, y algunos casos de estilo indirecto.

Los datos que aportamos son textuales en latín y tomados de la lengua hablada en inglés.

\section{COMPLEMENTACIÓN CON INFINITIVO}

En latín, a diferencia de lo que ocurre en inglés, se utiliza pocas veces el infinitivo como complemento de nombres (adjetivos o sustantivos), y los ejemplos de este uso, salvo los de la comedia, corresponden generalmente a los períodos postclásico y siguientes ${ }^{2}$.

\section{II.1. Infinitivo Complemento de Adjetivos}

Este uso, frecuente en inglés antiguo y moderno, es poco utilizado en la prosa clásica pero frecuente en los poetas de la época augústea y períodos subsiguientes, e igualmente en los prosistas poetizantes (debido, posiblemente, a la influencia griega ${ }^{3}$.

En latín presentan esta construcción adjetivos que admiten un infinitivo como régimen, construcción que se propaga a adjetivos que tienen valor absoluto $\mathrm{y}$, por tanto, no han de llevar necesariamente determinación por tener un sen-

2 Cf. Bassols De Climent, M.: Sintaxis Latina I, Madrid, 1967, pp. 358 y ss.; Bennet, Ch.E., Syntax of Early Latin, Hildesheim, 1966, pp. 417-8; RIEMANN, O.; Syntaxe latine, París, 1942, pp. 501 y ss.; LeUMANN, HofFMANN \& SZANTYR, Lateinische Grammatik, Zweiter Teil, pp. 350 y ss.

3 En griego se utiliza esta costrucción con adjetivos que expresan habilidad, aptitud facilidad etc. cf. O. Riemann et Ch. Cucuel, Syntaxe grecque, París, 1941, p. 194. 
tido completo, como celer, blandus, durus, horrridus, y ardus. Ilustramos algunos ejemplos:

$\begin{array}{ll}\text { celerem sequi } & \text { (Hor. Carm. 1,15,18) } \\ \text { horrida cerni } & \text { (Luc. 3,347) } \\ \text { arduum dignosci } & \text { (Val. Max. 6,8,5) }\end{array}$

Con bastante frecuencia, en latín, se utiliza, en lugar del infinitivo, el gerundio en genitivo dependiendo de adjetivos como cupidus, studiosus y peritus (cfr Hec. 283: cupidus redeundi domum; Cat. Frag. (Jord.) 78,13: colendi peritus). Estos adjetivos en inglés pueden utilizarse de forma absoluta o complementarse con infinitivo, pero no admiten complementación con gerundio.

En inglés, por su parte, se observan diferentes tipos de complementación de adjetivos. Algunos, preferentemente, utilizan el infinitivo, si bien pueden complementarse con otros medios, tales como oraciones completivas, gerundios, o, al igual que en latín, utilizarse solos.

En lo que se refiere a casos paralelos en ambas lenguas, encontramos adjetivos que indican actitud o valoración de una actividad humana; facilidad o dificultad; cualidades tales como capacidad, habilidad, rapidez o lentitud; y de contenido emotivo. Así, por ejemplo:

\begin{tabular}{|c|c|}
\hline 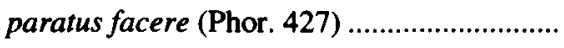 & ready to go \\
\hline 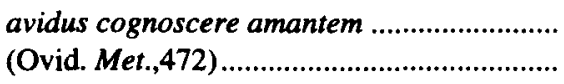 & $\begin{array}{l}\text { eager to help } \\
\text { anxious to knov }\end{array}$ \\
\hline solvere nulli lentus (Luc. 414) ........................ & slow to react \\
\hline $\begin{array}{l}\text { felix... ponere vitem } \\
\text { (Virg. Georg. } 1,284 \text { ) }\end{array}$ & happy to hear react \\
\hline $\begin{array}{l}\text { facilis spargi ...rosa } \\
\text { (Prop. } 4,8,40)\end{array}$ & easy to handle, \\
\hline
\end{tabular}

adjetivo este último que, en latín, puede complementarse en época tardía con gerundio en dativo. 


\section{II.2. Infinitivo Complemento de Sustantivos}

En latín tal clase de complementación no aparece hasta la época postclásica. En todas las lenguas germánicas este uso es bastante frecuente, y, en inglés, se extiende a casos que el latín no contempla. Ambas lenguas comparten sin embargo, algunas expresiones de esta estructura, como por ejemplo:

spes (the) hope (to...)

occassio (the) opportunity, chance (to...)

(PI. Pers. 725: nunc est illa occassio inicium ulcisci; PI. Capt. 423: nunc adest occassio bene facta cumulare)

modus .......................... (the) way (to...)

(Pl. Men. 233: quid modi illum quaerere?)

tempus (the) time (to...)

(Pl. Asin. 912: tempus est subducere hinc me)

potestas

(the) power (to...)

facultas

(the) faculty (to...)

(Bell. Afrc. 78,4: ut haberent facultatem...pugnare)

cupido (the) wish (to...)

(Enn. Scaen. 257: cupido cepit miseram nunc me proloqui)

Está claro que en muchos casos de estos ejemplos no siempre se podría delimitar si el verbo en infinitivo es complemento del sustantivo o del contenido semántico de la expresión de sustantivo más verbo, pues en muchos casos se recurre a los gerundios para dicha complementación, como en el caso de spes, potestas y otros.

Incluso en algunos textos los ejemplos no son seguros, ya que se trata de locuciones como tempus est, o bien consilium ceperunt, en las que la dependencia del infinitivo no serfa estrictamente del sustantivo sino del valor semántico de dicha perifrasis, como podemos observar en lo que sigue: 
(Cic.) ibi Pomponium expectare consilium est.

They made the decision to have a meeting in two months

\section{II.3. Infinitivo Complemento de Verbos}

En lo que se refiere a usos paralelos en este apartado, tanto una lengua como otra utilizan infinitivos para complementar verbos o expresiones impersonales, verbos auxiliares modales, así como verbos de lengua, entendimiento, percepción, voluntad, causativos y otros ${ }^{4}$.

II.3.1. En latín se utiliza el infinitivo para complementar una variedad de verbos impersonales (sentimiento, conveniencia, licitud, facilidad, placer, sucesos o similares, etc. ${ }^{5}$, y expresiones impersonales formadas, generalmente, con el verbo sum más adjetivo o sustantivo abstracto, incluso adverbio. Así, por ejemplo, en:

Men. 802: melius, sanam est mentem sumere

Plaut. Amph. 29: mirari non est aequom

Curc. 176: bonum est pauxillum amare

Enn. Trag. 132: (ita) sapere opino esse optumum

En inglés, en cambio, esta circustancia únicamente se da en expresiones impersonales, y no en verbos puramente impersonales que indican fenómenos atmosféricos y otros y en los que no cabe tal tipo de complementación. Algunos ejemplos:

4 Cf. Bassols, M., op.cit. Tomo I, pp. 349-354; BenNeTt, CH.E., op.cit., pp. 399-406; Leumann ..., op. cit., pp. 353 y ss.; Ernout, A. \& Thomas, fr., Syntaxe Latine, París, 1964, pp. 328 y ss.

5 Este uso es bastante más frecuente en latín decadente, ya que muchos verbos que en el período clásico no admitían este uso impersonal lo adpotan. 
It's neccessarry to revise some old, worn-out concepts

It's better to stay here and wait

It's a pity to spend so much money on that

Con todo, este uso en las expresiones impersonales podríamos considerarlo como una extrapolación del sujeto, que sería la propia oración de infinitivo.

II.3.2. Con muchísima frecuencia tanto el latín como en inglés el infinitivo funciona directamente como complemento del verbo en forma personal. Esta función recibe el nombre de «concertado» frente al «no concertado» que desempeñaría la oración de infinitivo propiamente dicha.

En latín, con los verbos modales (possum, debeo, soleo, assuesco, consuesco, etc.) únicamente encontramos el infinitivo en función de complemento directo y sin poder desempeñar otra distinta. En cambio, en una serie de verbos de volición: volo, nolo, malo,etc.; orden o mandato: iubeo; ruego o petición: peto, postulo; permisibilidad o consentimiento: sino, patior; y verbos que expresan una manifestación de la actividad, como cogo, faccio, etc., generalmente-salvo en los verbos de voluntad-la mayoría de las veces se prefiere la construcción de oración de infinitivo con sujeto propio y la de ut. Con todo, no faltan ejemplos, incluso en la prosa clásica, de cualquiera de estos verbos complementados con un simple infinitivo. Así, por ejemplo:

Plaut. Asin. 67:

Plaut. Bacch. 581:

T. Livio, XXII, 2,6:

T. Livio, XXII, 2,2:

T. Livio, XXII, 3,11 volo amari

fores pultare nescis

Galli neque sustinere...neque adsurgere po-

terant

primos ire iussut

cum ocius signa convelli iuberet.

Paralelamente, verbos ingleses pertenecientes a grupos similares se complementan también con infinitivo (con o sin «to», dependiendo de los casos), si bien algunos admiten asimismo la posibilidad de completiva introducida por that, así como la oración de infinitivo. Como en latín, se puden complementar con infinitivo todos los verbos modales, verbos de volición como want, refuse, desire, etc., de ruego o petición como ask, request; y de permisibilidad o consentimiento del estilo de condescend, consent, como muestran los siguientes ejemplos: 
I want to have a word with you

She asked to leave

Ican't tell the difference between green and blue.

\subsubsection{Oraciones de Infinitivo}

Este tipo de construcción ${ }^{6}$ ha sido objeto de numerosos estudios por parte de la linguiística contemporánea en su variante generativa a partir del célebre libro de R.T. Lakoff Abstract Syntax and Latín Complementation (Cambridge (Mass.): C.U.P.,1968), y al trabajo reciente de Calboli, Maraldi, Perrochat y Herman ${ }^{7}$.

En ambas lenguas consideramos Oración de Infinitivo la construcción de infinitivo con sujeto expreso en acusativo. (Debemos señalar que aunque no hay diferencia clara entre los dos tipos de construcción exire volo y te exire volo, hay que considerar que el mismo empleo del infinitivo no las hace equivalentes en su uso).

La oración de infinitivo depende de verbos de lengua, entendimiento y percepción, voluntad, causativos y de dirección (orden, ruego, recomendación), y puede, asimismo, desempeñar función de sujeto, en una expresión de las tenidas como impersonales del estilo de «licet»; en inglés, en construcciones con sujeto extrapolado y otras, pues aquéllas que se consideran puramente «impersonales» (referidas a fenómenos atmósfericos, naturales, etc.) no admiten tal tipo de complementación. En cualquier caso, y en las dos lenguas, el sujeto de la ora-

${ }^{6}$ El trabajo de Lakoff, R. T., Studies in the Transformational Grammar of Latin: The Complement System, 1968, supuso un gran avance para la ligüfstica al tiempo que llegó a confirmar (en el cápitulo tercero) que hay una relación bastante estrecha entre la estructura sintáctica del latín y la del inglés, y que las diferencias existentes entre ambas lenguas eran superficiales y de poca importancia.

7 Cf. otros trabajos interesantes para este apartado: CALBOLI, G., «The Developement of Latin (Cases and Infinitive)", en PINKster, H.(ed), Latin linguistic and linguistic Theory, Amsterdam; Jhon Benjamins, pp. 41-57; MARALDI, M., «New Approaches to Accusative Subjects: Case Tehory vs. Raising", en Pinkster, H. (ed.), op. cit., pp 167-176; Perrochat, P., recherches sur la valeur et l'emploi de l'infinitif subordonné en latin, París: Les Belles Lettres, 1932.

Cf. asimismo Herman, J., «Accusativus cum infinitivo et subordonee a quod, quia en latin tardif. Nouvelles Remarques sur vieux probléme». 
ción de infinitivo aparece en acusativo ${ }^{8}$ (en inglés, en el segundo tipo, el sujeto va introducido por «for»).

Ejemplos del primer uso los encontramos en:

volo te venire

iubeo te venire

T. Liv. XXII, 5,6:

I heard her scream ${ }^{9}$
I want you to come

I order you to come

apparruitque nullan nisi in dextra ferroque salutis spem esse

Algunas del segundo tipo de expresiones serían:

For you to say so is most inconvenient

It's essential for European countries to develop common policies on that matter

\section{ESTILO INDIRECTO}

El llamado Estilo Indirecto ${ }^{10}$ es una estructura sintáctica en la que las palabras o pensamientos de otra persona o propios son transmitidos bajo la forma de oración subordinada a un verbo o palabra con valor declarativo expreso o so-

${ }^{8}$ En esta afirmación debemos puntualizar que en la lengua latina encontramos algunos ejemplos de or. de infinitivo con sujeto en nominativo. Dicha construcción, propia del lenguaje poético y generalizada algo más a partir del período postclásico se debe posiblemente a las oraciones de infinitivo dependiendo de verbos de lengua y entendimiento con elipsis del sujeto y del predicado formulado en nominativo. En Hor. Epist. 1, 7.22: ait, esse paratus en lugar de ait se esse paratum Cf RiemanN, O. Op. cit. pp. 320 s.; Bassols, M., Op. cit. pp. $228 \mathrm{~s}$.

9 Estos verbos de percepción se construyen en inglés con infinitivos sin $t o$.

10 Tema muy debatido, existe gran abundancia de trabajos y opiniones sobre el mismo. Por lo que respecta a la lengua latina, la estuctura de estas expresiones quedó perfectamente establecida en el trabajo del prof. L. RuB10, Introducción a la Sintaxis Extructural del latin, vol. II, Barcelona, 1976, pp. 65-78, Al que hay que agradecer la claridad de exposición, y ante todo, la acertada, sabia y tajante separación que hace el autor entre estilo indirecto y subordinación. 
breentendido o una expresión de contenido similar (así como mediante otros giros que expresan el mismo contenido de lo que en su momento fuera expresión en estilo directo).

\section{III.I}

Entre latín e inglés hay una estrecha relación en cuanto a modos verbales en este tipo de construcciones, ya que, por lo general, se utilizan infinitivo y subjuntivo.

En oraciones no subordinadas, la lengua latina utiliza el infinitivo para espresar el contenido aseverativo, es decir el mensaje enunciativo; el subjuntivo, para el contenido impresivo, es decir, mensaje afectivo que indica deseo, orden y ruego, y finalmente, cualquiera de los dos modos en las interrogativas. En cuanto a las oraciones subordinadas, se utiliza normalmente el subjuntivo. Los textos que encontramos, principalmente formulados en infinitivo, son numerosísimos; aquí únicamente ofrecemos una muestra representativa de los dos tipos que se utilizan:

A) Aseverativo:

T. Liv. XXII, I, 8:

«Augebant metum prodigia ex pluribus simul locis nuntiata: en Sicilia militus aliquot spicula, in Sardinia autem in murro circumeunti vigilias equiti scipionem, quen manu tenuerit, arsisse...»

T. Liv. XXII, VI, 11:

«fidem dante Maharbale;..., si arma tradissent, abire cum singulis vestimentis passurum, sese dediderunt».

B) Impresivo:

Caes. B.C. I, 85, 12:

«Ad ea Caesar respondit:...Proinde, ut esse dictum, provinciis excederent exercitumque dimiterent; (sed) si id si factum, se nociturum nemini.» 
En inglés, por su parte, también son posibles ambos usos, el de la oración subordinada y el de la estructura de infinitivo. Por lo que respecta a este último, es frecuente en casos de estilo indirecto con verbos de lengua y con directivos, algunos de los cuales admiten ambas posibilidades (cf. «he promised that he'd come with us»/ «he promised to come with us», o «he said he was a member»/ «he said to be a member»), mientras que, para otros, la única estructura posible es la del infinitivo, como en «I told the children not to move».

\section{Conclusión}

Como hemos visto, los casos de identidad estructural en ambas lenguas en lo que se refiere a construcciones de infinitivo son numerosos, y de tal índole que hacen pensar en un desarrollo paralelo que quizá no sea casual.

No ha sido, sin embargo, nuestro objetivo desvelar a qué se deba que dos lenguas por lo demás tan dispares muestran tales y tantos casos de uso común de ciertas estructuras linguísticas. Nos ha parecido interesante destacar la existencia de tales casos, que puede tener su trascendencia incluso en el enfoque con que nos aproximemos, tanto en su estudio como en su enseñanza, a estas lenguas.

Desde este punto de vista podríamos entonces apreciar la importancia que para el estudio de ciertos idiomas modernos tienen lenguas clásicas como el latín, al tiempo que el acercamiento a estas últimas podría beneficiarse en gran manera de la utilización de metodologías que hasta la fecha han sido más propias de lenguas pertenecientes a cierto ámbito espacio-temporal que excluía el mundo de lo clásico. 